\title{
Design and Implementation of A Circuit Board Calibration System
}

\author{
Hang Bai ${ }^{1}$, Yunlai Huang ${ }^{1}$, Di Lu ${ }^{1}$, Ping Lai ${ }^{1}$ and Hongzhi Fan ${ }^{1}$ \\ ${ }^{1}$ Unit 63981 in Wuhan, China
}

\begin{abstract}
With the development of science and technology, the traditional artificial detection methods cannot meet the requirements of modern equipment testing and calibration. Combined with the actual demand, a kind of circuit boards calibration system are put forward. It can to realize automatic testing and calibration of the circuit boards. Many functions of the calibration system such as automatic testing, self-test and monitoring are summarized. The hardware is introduced which including the industrial computer system, calibration adapter and so on. Then, development platform, the thought of program design and the structure of the software are introduced in detail. The function of automatic calibration to specific circuit boards are realized. Because the system has good commonality and easy to extend to upgrade, the development ideas and experiences can be applied to similar circuit boards automatic testing system.
\end{abstract}

\section{Introduction}

With the improvement of production technology and process level, advanced technology of the circuit boards are popular, such as high density, advanced packaging. The demands of advanced testing of circuit boards are become increasingly higher. In the circuit boards test of past, a kind of manual circuit probe testing are required. For higher requirements to the operation of the tester, the manual test methods are low efficiency and the reliability is not high. In actual measurement, the test results are often be impacted due to the poor contact between line and line. It cause the wrong test results. Visibly, the traditional manual inspection maintenance means already cannot satisfy the requirement of the modern equipment support. Automatic test system is gradually becoming necessary to ensure reliable of complex systems and equipment operation. For the low efficiency of manual test and low reliability, the test methods are put forward combined with the actual demand in references. A circuit board calibration system is designed to meet all testing and calibration demand of the certain equipment circuit boards. It can save a lot of manpower material resources. It can also reduce the project cycle. At the same time, it can greatly improve the accuracy of the testing.

\section{System function}

Calibration system can realize automatic test and parameter calibration of all circuit boards for a device. It can guide to carry out fault diagnosis and maintenance of the circuit board. It has main functions as following.

\subsection{Automatic testing function}

The system can provide signals input and real-time working environment, according to the demand of the testing process. It can realize performance testing of the calibrated objects measuring the outputs of the response signals. The values of the signals are compared with the standard values. If the values are not normal, the initial fault diagnosis and maintenance advices are given.

\subsection{The self-test and monitoring function}

The system has good reliability and strong self-test functions. Before working, the main boards, calibration adapter and power supply are in the testing. The state of the adapter is monitoring in the testing process. When the voltage is abnormal, The hardware of the adapter reset immediately to ensure the safety of calibration system and calibrated objects. It will give an alarm until artificial lift fault.

\subsection{Friendly man-machine interface and function of human intervention}

A good operating environment is constituted by using a graphical engineering language and a graphical interface. It provides a comprehensive help for testing and failure analysis, and the connection is simple, easy to operate. When the calibration system is working, the testing data can be stored at the same time. After completion of the test, the measurement data is processed automatically and the calibration certificate is generated. Users can query historical test data at any time according to actual needs. 


\section{The system hardware design}

The advanced and mature computer technology, automatic calibration technology, microelectronics technology, VXI bus technology and virtual instrument technology are used to realize automatic testing and fault diagnosis in the system. The main functions of the system hardware are provide working conditions for calibration object and exert all kinds of excitation signal. By gathering its response and judging of its performance, it can provide information for fault diagnosis. According to the characteristics and requirements of the calibration object, the system hardware is designed. At the same time, it can meet the whole system design requirements on the technology of modularization, generalization, intelligent, standardization. As shown in fig 1, the design of the hardware mainly consists of industrial computer system, calibration adapter and test cable.
Industrial computer system is the core of the calibration system, including the industrial PC and data collection module, communication module and signal control module. The main function of the system is to complete testing task scheduling and management, testing process control and testing response analysis, testing results show, report generation, database management, etc. Calibration adapter is the key to the system. It is consist of test interface relay combination, programmable power supply, etc. It provides all kinds of excitation source and interface for calibration object. These excitation sources will arrive the corresponding ports. Calibration system involves many different interface circuit boards. The pin signal characteristics of these interfaces also each are not identical. Therefore, it need to design interface conversion circuit.

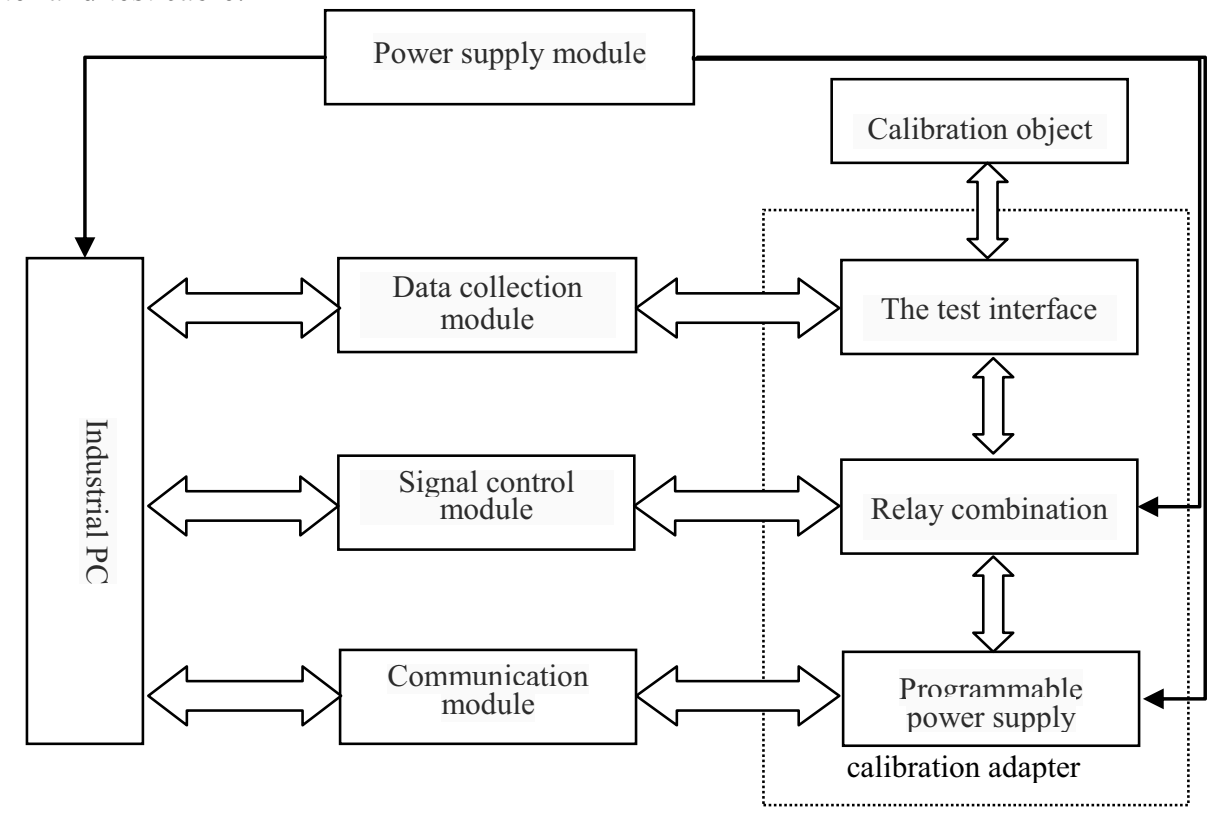

Fig 1.The hardware composition of the system

The interface conversion circuit includes interface circuit and relay interface circuit part. Different interface pins connect to a unified interface. The signals pins are switched by relay assembly. In order to realize the automatic control of the test objective, the relay assembly realize the switching of different input signal and output signal. It works according to different calibration object. Programmable power supply can provide 8 way power and signal source output. It provides the actual working conditions for the calibrated object. It also has many advantages, such as strong anti-interference ability, independence, good interchange ability, high precision and so on. The system detection circuit is mainly convert each channel power supply current to voltage. The output of voltage is connected to the analogy input module. Real-time voltage and limit value are compared by the comparator. when any channel current exceed the limit value, sending a high level to relay the total voltage control relay. It forced shutdown relay total voltage. The main power and the calibration object will be isolation and incentive. It reset the hardware to protect the calibrated object and system security.

\section{The system software design}

\subsection{Software development platform}

With the improvement of the performance of the PC and the reducing of the price, developing testing software based on Windows platform has become a trend. Because the data processing is relatively complex and good human-machine interface is required, the Visual Studio 2010 is chosen as the development platform, using C\# language to develop the automatic testing software.

\subsection{The program design thought}

When calibration software is designed, The functions are realized from top-down based on the calibration process. All of the testing parameters, programmed instructions 
and testing results are placed inside the testing software. It enhances the reliability of the software system. The using of powerful interface function and reasonable construction of the interface can analyze the testing data scientifically. The use of powerful data processing technology can analyze and calculate the testing data. The electrical performance parameters of the objects at work can be gotten as accurately as possible. According to the system objectives and requirements, the software system, its interface technical index and function requirements are constructed reasonably using modular, universal and object oriented design thought. Efficient development, using and maintenance of software is realized.

For different testing and calibration of tasks, software executive function is different, but the process is similar. Each testing and calibration tasks can be decomposed into a number of test and calibration unit. Each unit can complete a test. The test data are analyzed. So that you can complete the board testing and calibration performance by a subroutine. According to the specific situation of testing and rectification of the object, testing initialization function and calibration function are written respectively. Because many parameters need to be tested and revised, it involves many interface. Combined with the hardware platform having a great room for developing, it need to write a program management interface. The interface can realize scientific management for the calibration procedure. It can also establish the necessary interface specification for subsequent program development. As for the self checking module and parameter adjustment module of the calibration system, it need to write the control function respectively. It can call for the control function any time according to the calibration needs. The calibration object and debugging process not only need to be considered in the process of compiling, maximize function of the interface circuit and adapter, but also give a convenient interface for the subsequent development of the program.

\subsection{The system software structure}

Calibration software includes two parts. One is the system software and the other is application software. System software is the operating system of the computer and test software development environment. It is used for computer software development. Application software is the calibration program, used to configure and control test platform hardware resources. Signal acquisition, data processing, data report and fault diagnosis are completed according to the calibration request. The calibration of the system software structure as shown in Fig 2.

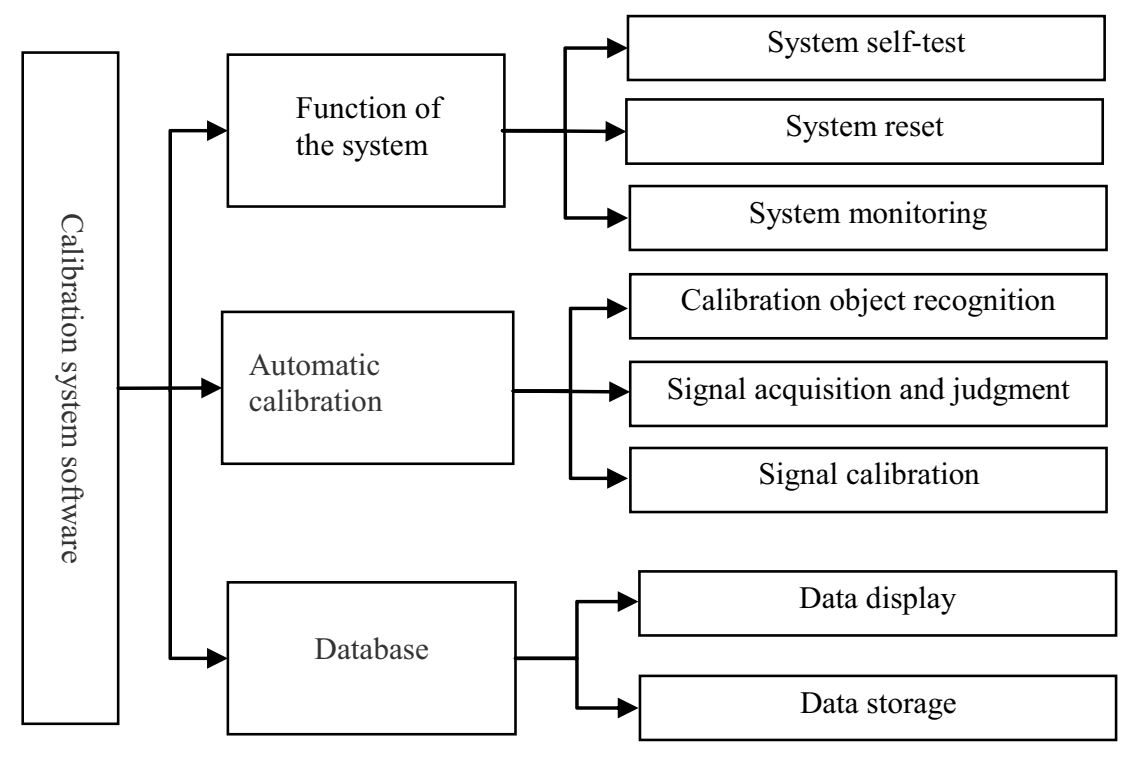

Fig 2. Structure of system software

The function of the calibration software includes the selftest, reset and monitoring. The self-test system mainly start relay assembly self checking circuit through the signal control module programming. The channel adapter power transfer to the analogy input module. By judging the power adapter and relays the self-test results to determine whether it pass self inspection and display. If failed, it can be unable to carry out other operations to ensure the safety of equipment and system. The system reset means that signal control module will be zero clearing to reset all relays. The system reset must be carried out before or after calibration to ensure the calibrated object security. The main function of the monitoring system throughout is to monitor the main channel power and real-time excitation current. The software process is as a background thread.

Automatic calibration module is the core part of the circuit board calibration software design. It is the circuit board single board automatic testing effective working module software design. The functions of the module are as following. Firstly, automatic recognition and detection of whether the object is a circuit board can be detected, and can detect when a call in a calibration function for calibration. Secondly, it can calibrate the circuit board and get the results of the analysis and judgments. Thirdly, it can analyze and locate the fault positioning of the circuit board. The automatic testing software only need to follow the steps required for programming analogy input 
module, signal control module and serial communication module. It will be able to judge the calibration object technical condition by judge the analogy input voltage corresponding to the channel module collected. Access 2000 database is used as the data collecting platform. Database collecting platform takes the task of managing calibration configuration information, resource information, calibrated object information, the results of data analysis and records.

\section{Conclusion}

The circuit board calibration system is mainly used for the calibration of parameters of the certain type of equipment all circuit boards. It can assess function and indexes of the circuit board whether qualified. The system has the advantages of fast testing ability strong. It have achieved good results in practical application. As long as a few modifications to the system can realize the functions and the new requirements, which can meet the needs of different types of automatic circuit board testing.

\section{References}

1 HU Kunlin, LI Ye, ZUO Xiangli, et al. Design of a virtual logic analyzer with multi-channels based on LabVIEW and FPGA[J]. Application of Electronic Technique, 2012,38(11):91-93.

2 Hua San, He Liming, Tian Zuohua. Design and Realization of Circuit Board Auto-Test System [J]. Measurement and control technology, 2008.07(27)15-19.

3 LIU Ying. Design on Circuit Board Auto Test System Based on Virtual Instrument [J]. Computer \& Network, 2010.02:46-48.

4 Wang Chunyou, Lian Guangyao, Wang Zhensheng, Liu Yanhong. Design and Realization of Portable ATS for PCB [J]. Computer Measurement \&Control, 2009.17(12)2407-2409.

5 WANG Zhaofeng, WANG Shicheng, MU Jianhua, ZHANG Dayong. Implementation of the Automatically Calibration and System for the Missile's VXI Measurement System [J]. Journal of missiles and guidance, 2005.04(25)389-390.

6 WANG Xiaoliang, XIE Zhaohui, YANG Hongguan. A new memory testing system based on LabVIEW[J]. Computer Measurement \& Control, 2012,20(7): 1763-1765.

7 Xue Jiapu, Su Lei, Chen Yong, Li Yunfei . Development of controller for a circuit board testing system [J]. TECHNOLOLOGY TREND, 2010.18(11):91-92.

8 Zhang Jingwei, Li Ruozhong, Wang Dewang. Study on Test System of Missile Equip PCB Based on Expert System [J]. Computer Measurement \&Control, 2012.20(11)3005-3007. 